Alonso, Julia; Román, José-María (2014). Nivel sociocultural, prácticas educativas familiares y autoestima de los hijos en edades tempranas. Revista de Investigación Educativa, 32 (1), 187-202.

http://dx.doi.org/10.6018/rie.32.1.173421

\title{
NIVEL SOCIOCULTURAL, PRÁCTICAS EDUCATIVAS FAMILIARESY AUTOESTIMA DE LOS HIJOS EN EDADES TEMPRANAS
}

\author{
Julia Alonso y José-María Román \\ Universidad de Valladolid
}

\section{RESUMEN}

Los trabajos empíricos que relacionan nivel sociocultural de las familias con autoestima de los hijos en edades tempranas son escasos. Aun más escasos son los que tienen en cuenta la percepción infantil de las "prácticas educativas" del padre y de la madre en edades tempranas. Dos trabajos - realizados con un año de intervalo, replicación directa uno del otro - relacionan prácticas educativas familiares y autoestima en familias de nivel sociocultural alto, uno (Alonso E Román, 2005a) y prácticas educativas familiares y autoestima en familias de nivel sociocultural bajo, otro (Alonso y Román, 2005b). Sin embargo, estaba sin hacer -objeto de este artículo - la comparación de ambos trabajos tomando el nivel socio-cultural de las familias como variable independiente asignada y las prácticas educativas y la autoestima de los hijos pequeños como variables dependientes asignadas.

Este artículo compara los resultados de esos dos trabajos independientes, realizados con familias de niveles socioculturales medio/alto (283 familias y 283 hijos) y medio/bajo (142 familias y 142 hijos).

Se constata que el nivel sociocultural de las familias condiciona: (a) la diferente percepción que de las prácticas educativas familiares tienen padres e hijos, (b) el nivel de concordancia entre ambas percepciones y (c) la distinta influencia en la autoestima de los hijos.

Palabras clave: nivel sociocultural familiar; prácticas educativas familiares; autoestima de hijos pequeños.

\footnotetext{
Correspondencia:

Departamento de Psicología. GIE (GR179) en Psicología de la Educación. Facultad de Educación.

Universidad de Valladolid. Campus “Miguel Delibes”. Paseo de Belén, 1: 47011 Valladolid.

Email: jalonsog@psi.uva.es y/o jmroman@psi.uva.es
} 


\section{SOCIOCULTURAL LEVEL, FAMILY EDUCATIONAL PRACTICES AND SELF- ESTEEM OF YOUNG CHILDREN}

\section{ABSTRACT}

Empirical research on the relation between sociocultural level of families and self-esteem of children at an early age is scarce. Even rarer are those which take into account the child's perception of his/her father's or mother's "educational practices" at an early age. Two studies (one is the replication of the other) analyzed the relation between family educational practices and self-esteem in high sociocultural level families (Alonso and Roman, 2005a), and family educational practices and self-esteem in low sociocultural level families (Alonso and Roman, 2005b). This article made the comparison of both studies taking family sociocultural level as an independent variable, and educational practices and self-esteem of young children as dependent variables. The results showed that sociocultural level determined: (a) differences in perceptions of family educational practices held by parents and children, $(b)$ the level of agreement between both perceptions, and (c) the influence on children's self-esteem.

Keywords: family sociocultural level; family educational practices; self-esteem of young children.

Numerosos trabajos de investigación han tratado de analizar el grado de asociación entre el nivel sociocultural familiar y distintos aspectos del desarrollo de los hijos, el logro académico (Calvete, Orue \& Sampedro, 2011, o Weiland \& Yoshikawa, 2012) o la conciliación familiar (Fraguela, Lorenzo \& Varela, 2011). Se le ha relacionado con la salud física y psíquica, con problemas de atención o trastornos de la conducta tales como la agresividad o el acoso, el rendimiento escolar o el absentismo. También hay trabajos que lo relacionan con el clima familiar, el bienestar psicológico, las prácticas de crianza o la eficacia de los programas de intervención parental (Ondersma, 2002; Soriano \& Franco, 2011).

Sin embargo, es difícil encontrar trabajos que relacionen el nivel sociocultural familiar y las prácticas educativas - auto y hetero-percibidas - de padres y madres. En un trabajo de Alonso \& Román (2005a) hay un ejemplo de ello. Menos frecuente aun es tener en cuenta la percepción infantil de las prácticas educativas de sus padres; y mucho menos, estudiándolas en edades tempranas. En dos trabajos de Alonso \& Román $(2005 a, b)$ se hace esto. Son una replicación directa con familias de nivel sociocultural diferente: medio/alto y medio/bajo.

La carencia de estudios coincidentes y el interés por identificar el impacto bidireccional, desde las primeras edades, en los contextos familiares de distinto nivel sociocultural, justifica el interés por realizar un estudio comparativo entre esos dos trabajos previamente publicados. Ambos estudios realizan un análisis pormenorizado de las interacciones entre padres, madres, hijos e hijas de 3, 4 y 5 años pero de diferente nivel sociocultural.

Común en ambos trabajos era analizar la correlación - o grado de asociación - entre las prácticas educativas familiares y la autoestima de los hijos. Lo diferente en ambos es que los datos provienen de dos muestras de distinto nivel sociocultural (medio/alto y medio/bajo), recogidos con un intervalo de un año. 
La posible correlación o asociación entre el nivel de autoestima de los hijos y las prácticas educativas de los padres - o estilo educativo predominante - (" $\mathrm{r}$ " positiva con las autorizadoras o equilibradas y " $\mathrm{r}$ " negativa con las prácticas autoritarias y permisivas), es una hipótesis común en el primero (nivel sociocultural medio/alto) y segundo trabajo (nivel sociocultural medio/bajo).

En el segundo estudio (replicación directa del primero, con familias de nivel sociocultural medio-bajo) pretendieron - además - verificar otras hipótesis, tales como: (a) si los padres y madres utilizarían con mayor frecuencia los estilos educativos equilibrado y permisivo en situaciones en que sus hijos inician algo nuevo; (b) si los adultos utilizarían indistintamente los tres estilos educativos en situaciones en que sus hijos cuentan o muestran algo; (c) si es en ambas situaciones donde la percepción del estilo educativo familiar tendría un mayor nivel de discrepancia entre padres, madres e hijos; (d) si tendrían un mayor nivel de acuerdo adultos e hijos percibiendo estilo educativo autoritario y/o permisivo en situaciones en que sus hijos realizan ruptura de rutinas; (e) si los padres y madres utilizarían indistintamente cualquiera de los tres estilos educativos en la resolución de los conflictos externos; (f) si en la resolución de los conflictos internos, es donde la percepción del estilo educativo familiar sería de mayor permisividad; (g) si en la resolución de conflictos de transgresión de normas, es donde la percepción del estilo educativo autoritario tendría un mayor nivel de acuerdo entre padres, madres e hijos.

Así pues, el objetivo de este artículo es comparar ambos trabajos - cosa que no se ha hecho- e identificar si el nivel sociocultural de las familias está asociado, y en qué grado, - por un lado- con las prácticas educativas, y - por otro- con la autoestima de los hijos en edades tempranas.

\section{MÉTODO}

\section{Participantes}

Primer trabajo: Entre maestras, padres, madres e hijos participaron 1.132 personas, (tabla 1). Para seleccionar la muestra se utilizó la técnica "bola de nieve". Esta técnica de muestreo proporciona muestras muy homogéneas. El nivel instruccional de padres y madres de nivel sociocultural-medio/alto puede verse en la tabla 3.

TABLA 1

PARTICIPANTES EN EL PRIMER TRABAJO: NIVEL SOCIOCULTURAL MEDIO/ALTO

\begin{tabular}{|l|c|c|c|c|c|c|c|c|c|}
\hline \multicolumn{4}{|c}{ Maestras } & Padres & \multicolumn{1}{c|}{ Madres } & \multicolumn{1}{c|}{ Niños } \\
\hline & & $\begin{array}{c}\text { Edad } \\
\text { media: } \\
36 \text { años }\end{array}$ & $\begin{array}{c}\text { Edad } \\
\text { Media: } 34 \\
\text { años }\end{array}$ & $\begin{array}{c}3 \\
\text { años }\end{array}$ & $\begin{array}{c}4 \\
\text { años }\end{array}$ & $\begin{array}{c}5 \\
\text { años }\end{array}$ & $\begin{array}{c}3 \\
\text { años }\end{array}$ & $\begin{array}{c}4 \\
\text { años }\end{array}$ & $\begin{array}{c}5 \\
\text { años }\end{array}$ \\
\hline N Específicas & 283 & 283 & 283 & 56 & 55 & 33 & 63 & 41 & 35 \\
\hline N Global & 1132 & & & & & & & & \\
\hline
\end{tabular}


Segundo trabajo: 426 personas: 142 familias con nivel sociocultural-medio/bajo, sus hijos e hijas y las correspondientes maestras (tabla 2). La selección de la muestra se realizó mediante la técnica de muestreo aleatorio: único requisito para participar, la voluntariedad. Para garantizar la homogeneidad del nivel sociocultural y la heterogeneidad de núcleos familiares, el muestreo se realizó en dos centros escolares públicos de Madrid.

TABLA 2

PARTICIPANTES DEL SEGUNDO TRABAJO: NIVEL SOCIOCULTURAL MEDIO/BAJO

\begin{tabular}{l|c|c|c|c|c|c}
\hline \multicolumn{3}{|c}{ Familias } & \multicolumn{2}{c}{ Maestras } & \multicolumn{3}{c}{ Niños } \\
\hline & & & 4 años & 5 años & 4 años & 5 años \\
\hline & & & 32 & 34 & 39 & 37 \\
\hline N Especificas & 142 & 142 & 66 & & 76 & \\
\hline N Global & 426 & & & & & \\
\hline
\end{tabular}

En tabla 3, el nivel instruccional de padres y madres de ambos estudios. El segundo estudio, contó con un $63 \%$ de padres y mdres con estudios elementales, pero era aún menor, si se tiene en cuenta que los hijos estaban, preferentemente, a cargo de los abuelos, por motivos laborales de los progenitores. En el segundo estudio se tuvieron que eliminar los chicos y chicas de tres años para garantizar que comprendían los instrumentos de medida.

TABLA 3

NIVEL INSTRUCCIONAL DE LOS PARTICIPANTES

\begin{tabular}{|c|c|c|}
\hline Estudios & Trabajo-1 & Trabajo-2 \\
\hline Elementales & $32, \%$ & $63 \%$ \\
\hline Medios & $34 \%$ & $26 \%$ \\
\hline Superiores & $34 \%$ & $11, \%$ \\
\hline
\end{tabular}

\section{Instrumentos}

En los dos trabajos se utilizaron los mismos instrumentos para definir operativamente las tres variables. El nivel sociocultural de las familias se indujo a partir del nivel instruccional de los progenitores.

Las prácticas educativas familiares se evaluaron con las PEF-Escalas de identificación de prácticas educativas familiares (Alonso \& Román, 2003). La escala para padres y madres consta de cinco grupos o categorías de situaciones hipotéticas - consideradas relevantes y frecuentes por los expertos - ante las que los padres tienen que actuar:

1. Iniciar algo nuevo

2. Ruptura de rutinas

3. Ir a la cama

4. Contar o mostrar algo

5. Juegos 
A su vez, cada situación hipotética cotidiana, incluye tres tipos de conflictos:

a. Externo, circunstancias provocadoras del conflicto extrínsecas al hijo.

b. Interno, circunstancias provocadoras del conflicto intrínsecas al hijo.

c. Transgresión o incumplimiento de normas.

Para identificar el modo más frecuente de reaccionar del padre y/o la madre ante cada situación hipotética, se le ofrecen seis respuestas diferentes - dos indicadoras de prácticas educativas autoritarias, dos indicadoras de prácticas educativas autorizadoras o equilibradas y dos indicadoras de prácticas educativas permisivas. Se debe indicar la frecuencia con que suele responder así (figura 1).

Los sesgos y fuentes de error en las respuestas (engaño consciente, deseabilidad social y estilo de tendencia), se controlaron enfrentando la información de padre y madre (qué cree que haría su esposo o esposa y qué haría usted).

\section{SITUACIÓN HIPOTETICA-1: INICIAR ALGO NUEVO}

(a) CONFLICTO EXTERNO. Sabes que tu hijo o hija a esta edad, ya debe aprender a vestirse solo o sola. Imagínate que hoy estrena una camisa nueva y le cuesta abrocharse los botones. Protesta, se queja y pide tu ayuda para abrocharlos.

\begin{tabular}{|c|c|c|c|c|c|c|}
\hline ¿Con qué frecuencia crees que actuarías así...? & $\begin{array}{c}0 \\
\text { Nunca }\end{array}$ & $\begin{array}{c}1 \\
\text { Pocas } \\
\text { veces }\end{array}$ & $\begin{array}{l}2 \\
\text { Algunas } \\
\text { veces }\end{array}$ & $\begin{array}{c} \\
\text { Bastantes } \\
\text { veces }\end{array}$ & $\begin{array}{c}4 \\
\text { Muchas } \\
\text { veces }\end{array}$ & $\begin{array}{c}5 \\
\text { Siempre }\end{array}$ \\
\hline $\begin{array}{l}\text { 1. Pensaría que si le ayudamos aprendería a } \\
\text { depender excesivamente de nosotros; debe } \\
\text { hacerlo solo o sola. }\end{array}$ & & & & & & \\
\hline $\begin{array}{l}\text { 2. Ya que los botones tienen más dificultad, } \\
\text { y es la primera vez, le enseñaría y en cuanto } \\
\text { viera que pone interés le animaría y ayudaría } \\
\text { a terminar. }\end{array}$ & & & & & & \\
\hline $\begin{array}{l}\text { 3. Si lo hace habitualmente y ese día dice que } \\
\text { son más difíciles, no lo pondría en duda y se } \\
\text { los abrocharía yo. }\end{array}$ & & & & & & \\
\hline $\begin{array}{l}\text { 4. No vería la necesidad de ayudarle. Si todos } \\
\text { los días lo hace posiblemente sean caprichos y } \\
\text { no se le pueden consentir. }\end{array}$ & & & & & & \\
\hline $\begin{array}{l}\text { 5. Si protesta es que tiene razón y serán más } \\
\text { difíciles de abrochar así que le abrocharía yo } \\
\text { sin necesidad de perder el tiempo en discutir. }\end{array}$ & & & & & & \\
\hline $\begin{array}{l}\text { 6. Primero comprobaría si tiene razón y si es } \\
\text { así le enseñaría para que pudiera irlo haciendo } \\
\text { solo o sola poco a poco; si es necesario le } \\
\text { ayudaría. }\end{array}$ & & & & & & \\
\hline
\end{tabular}

FIGURA 1

UNA MUESTRA DE LA ESCALA A2 (ADULTOS) DE LAS PEF-ESCALAS DE IDENTIFICACIÓN DE "PRÁCTICAS EDUCATIVAS FAMILIARES". CLAVES: PRÁCTICA EDUCATIVA AUTORITARIA $(1,6)$. PRÁCTICA EDUCATIVA AUTORIZADORA $(3,5)$. PRÁCTICA EDUCATIVA PERMISIVA $(2,4)$ 
Las PEF son una adaptación de la tipología clásica de los estilos educativos de Baumrind (1971): Estilo autoritario: conjunto de prácticas parentales inflexibles y poco empáticas donde se da prioridad al control y al cumplimiento de las normas. Estilo autorizador, denominado equilibrado en las escalas PEF: conjunto de prácticas o maneras de actuar con los hijos que conjugan de forma equilibrada el control y la expresión de afecto y competencia comunicativa, en función de las características individuales y situacionales. Estilo permisivo: conjunto de prácticas o maneras de actuar con los hijos cargadas de mucho afecto, pero sin control y ni exigencia de cumplimiento de las normas ("dejar hacer"). El estilo negligente no se tuvo en cuenta debido a la escasa presencia del mismo en la cultura de las muestras de los dos estudios.

Las escalas PEF para los hijos tienen la misma estructura que las de los adultos pero simplificada. Tienen un soporte gráfico que facilita la comprensión de los hijos en edades tempranas (3, 4 y 5 años). La suma de las respuestas en cada situación y estilo -igual que en Escala A2 (adultos) - es la valoración cuantitativa de las prácticas educativas familiares. A mayor puntuación, mayor permisividad, y a menor puntuación, mayor autoritarismo. En la figura 2 puede verse un ejemplo de una de las situaciones y los tres tipos de conflicto de la Escala H2 (hijos) para aplicación infantil mediante entrevista.

\section{SITUACIÓN HIPOTETICA-1: INICIAR ALGO NUEVO}

(a) CONFLICTO EXTERNO. "Imagínate que hoy estrenas una camisa y te cuesta abrochar los botones. Protestas, te quejas y pides ayuda para abrocharlos"

\begin{tabular}{|l|l|l|l|}
\cline { 2 - 4 } \multicolumn{1}{l|}{} & $\begin{array}{l}\text { ¿Qué haría tu papá? ¿Qué haría tu mamá? (elige una de las tres opciones en } \\
\text { cada caso }\end{array}$ & Padre & Madre \\
\hline 1. & Te diría que lo tienes que hacer tu solo o sola & & \\
\hline 2. & Si ve que tienes razón te enseñaría a hacerlo. & & \\
\hline 3. & No lo dudaría y te los abrocharía & & \\
\hline
\end{tabular}

(b) CONFLICTO INTERNO. "Imagínate que eres muy tímido o tímida y que tienes que actuar al día siguiente en una obra de teatro del colegio. Es la hora de ir a dormir, pero estás tan nerviosa o nervioso que te cuesta ir a la cama"

\begin{tabular}{|l|l|l|l|}
\cline { 2 - 5 } \multicolumn{1}{c|}{} & $\begin{array}{l}\text { ¿Qué haría tu papá? ¿Qué haría tu mamá? (elige una de las tres opciones en } \\
\text { cada caso) }\end{array}$ & Padre & Madre \\
\hline $4 . \quad$ & Te mandaría a la cama pero te acompañaría un poco. & & \\
\hline $5 . \quad$ Te diría que tienes que ir a la cama como todos los días. & & \\
\hline 6. & Te diría que no hicieses la representación teatral. & & \\
\hline
\end{tabular}


(c) TRANSGRESIÓN DE NORMAS. "Imagínate que coméis fuera de casa y por primera vez vas comer algo que es muy difícil de pinchar con el tenedor. Como te gusta mucho, decides hacerlo con las manos"

\begin{tabular}{|l|l|l|l|}
\cline { 2 - 4 } \multicolumn{1}{l|}{} & $\begin{array}{l}\text { ¿Qué haría tu papá? ¿Qué haría tu mamá? (elige una de las tres opciones en } \\
\text { cada caso }\end{array}$ & Padre & Madre \\
\hline 7. & Te obligaría a comer con el tenedor. & & \\
\hline 8. & Ese día te daría de comer. & & \\
\hline 9. & Te enseñaría y te ayudaría. & & \\
\hline
\end{tabular}

FIGURA 2

UNA MUESTRA DE LA ESCALA H2 (HIJOS) DE LAS PEF-ESCALAS DE IDENTIFICACIÓN DE "PRÁCTICAS EDUCATIVAS FAMILIARES". CLAVES: PRÁCTICA EDUCATIVA AUTORITARIA $(1,5,7)$. PRÁCTICA EDUCATIVA AUTORIZADORA $(2,4,9)$. PRÁCTICA EDUCATIVA PERMISIVA $(3,6,8)$.

La autoestima de los hijos se midió con dos instrumentos. El primero lo hacía desde el punto de vista del propio niño (autopercepción) y el segundo desde el punto de vista de los padres y/o maestros (inferencia a partir de la observación conductual).

El PAI-Percepción del Autoconcepto Infantil de Villa \& Auzmendi (1999) se aplicó de forma individual mediante entrevista. Identifica el concepto que los niños de educación infantil tienen de sí mismos en estas diez dimensiones (autopercepción):

1. Autonomía: sentimiento de independencia.

2. Seguridad: confianza en uno mismo en la realización de las tareas.

3. Deportes: valía en la competición.

4. Familia: cómo se siente en su relación con la familia.

5. Aula: cómo se siente en el mundo escolar.

6. Social: relaciones sociales.

7. Sentimientos afectivos: estados de ánimo generales.

8. Autovalía: sentido de la propia competencia.

9. Aspecto físico: cómo valora su apariencia física.

10. Sentimientos de posesión: personas y objetos.

La BSECH-The Behavioral Rating Scale of Presented Self-esteem in Young Children de Haltiwanger \& Harter (1988), infiere el nivel de autoestima a partir de la conducta observada en casa y en la escuela. Explora con quince items estas cinco dimensiones :
a. Preferencia por los retos.
b. Iniciativa/independencia.
c. Preferencia social/retraimiento.
d. Expresión socio-emocional.
e. Forma de afrontar el estrés. 


\section{Procedimiento}

En el primer estudio se hicieron cinco valoraciones familiares: autopercepción del estilo educativo del padre, autopercepción del estilo educativo de la madre, heteropercepción del uno sobre el otro y heteropercepción del hijo sobre los dos. En el segundo trabajo solo se realizó una autopercepción adulta y la heteropercepción infantil. Esto se explica porque, por una parte, en contextos de niveles socioculturales más bajos es frecuente que el padre esté ausente - por razones laborales - y, por otra, debido a la necesidad de mantener la homogeneidad de la muestra, de nivel sociocultural-bajo, se eliminaron del estudio estructuras familiares no convencionales (no nucleares).

En cada familia de forma individual - investigadores entrenados - ayudaban a padres, madres, maestras y niños, a aplicar los cuatro instrumentos de recogida de datos: Escalas A2 (adultos) y H2 (hijos) de las PEF; PAI (Autopercepción infantil) y BSECH (heteropercepción conductual desde la familia y la escuela).

En este artículo se comparan - no hecho nunca - los resultados obtenidos considerando el nivel sociocultural como variable independiente asignada con dos categorías (alto y bajo) y las prácticas educativas familiares con tres categorías (autoritarias, autorizadoras y permisivas) y el auotoconcepto infantil (variable continua) como variables dependientes asignadas.

\section{Resultados}

(a) Nivel sociocultural de las familias y prácticas educativas de padres y madres.

Se observa en padres y madres, la tendencia a percibir como propio el estilo educativo que más valora el grupo sociocultural al que pertenecen. Especialmente en el

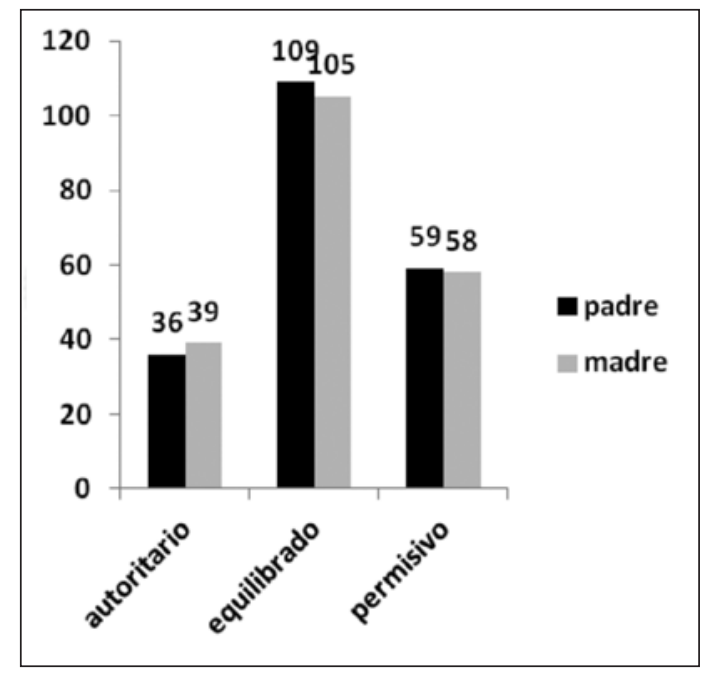

FIGURA 3

NIVEL SOCIOCULTURAL MEDIO/ALTO: AUTO-PERCEPCIÓN DE PADRES Y MADRES DE SU ESTILO EDUCATIVO. PUNTUACIÓN MÍNIMA 0, MÁXIMA 150. 
nivel sociocultural más alto, donde además coinciden en la valoración cónyuges e hijos. La figura 3 muestra la diferente autopercepción que padres y madres de nivel sociocultural medio-alto tienen de los estilos educativos.

En cambio, en la figura 4 puede verse la diferente autopercepción que las familias (solo contestó - por las razones antes citadas - un miembro de cada familia) de nivel sociocultural medio-bajo tienen de los estilos educativos.

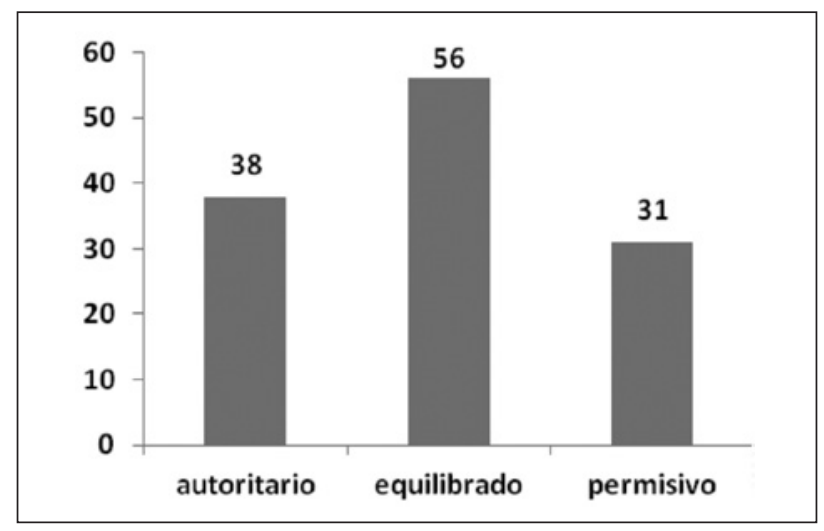

FIGURA 4

NIVEL SOCIOCULTURAL-MEDIO/BAJO: AUTO-PERCEPCIÓN DE PADRE O MADRE DE SU ESTILO EDUCATIVO. PUNTUACIÓN MÍNIMA 0, MÁXIMA 90.

En ambos niveles socioculturales se observa la utilización de prácticas educativas diferentes, en función del tipo de conflicto en que se encuentra su hijo y de las diferentes situaciones hipotéticas cotidianas (figura 5). No obstante...

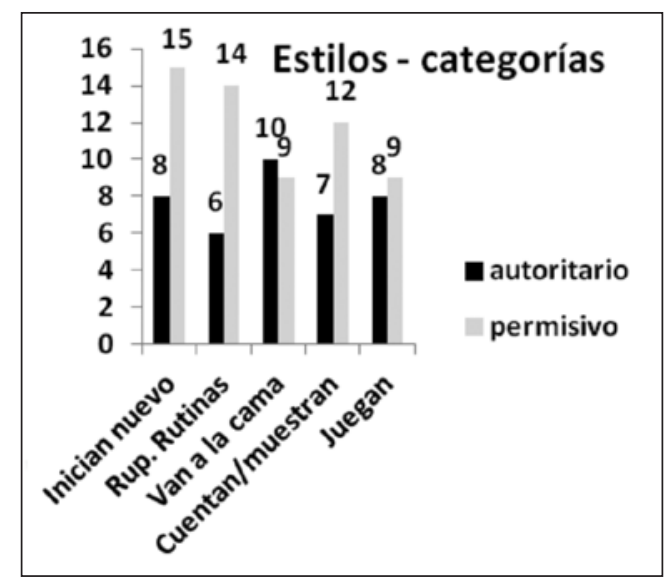

FIGURA 5

PADRE: COMPARACIÓN DEL ESTILO AUTORITARIO Y PERMISIVO EN LOS CINCO GRUPOS O CATEGORÍAS DE SITUACIONES HIPOTÉTICAS. PUNTUACIÓN MÍNIMA 0, MÁXIMA 30. 
En las familias de nivel sociocultural-bajo se observa una correlación o asociación significativa estable entre las distintas formas de medida de los estilos educativos parentales y el nivel de autoestima de los hijos (la " $\mathrm{r}$ " de Pearson indica el grado de asociación entre dos variables y la " $\mathrm{p}$ " la probabilidad de que esa asociación se deba al azar). Concretamente:

a) El nivel de autoestima global (autopercepción con el PAI y heteropercepción conductual de maestras y familias con la BSECH) correlaciona de forma positiva y significativa con el estilo educativo equilibrado o autorizador percibido por los hijos $(\mathrm{r}=.19 ; \mathrm{p} \leq .05)$; y de forma negativa y significativa $(\mathrm{r}=-.65 ; \mathrm{p} \leq .05)$ con el estilo educativo autoritario, percibido por los hijos.

b) El nivel de autoestima autopercibido (a través del PAI), correlaciona con el estilo equilibrado, percibido por el hijo, de forma positiva $(\mathrm{r}=.51 ; \mathrm{p} \leq .05)$; con el estilo autoritario, percibido por el hijo, de forma negativa $(\mathrm{r}=-.43 ; \mathrm{p} \leq .05)$; y con el estilo permisivo, percibido por el hijo, de forma negativa $(r=.-32 ; p \leq .05)$.

c) El nivel de autoestima, medido conductualmente a partir de la observación de la familia, correlaciona con el estilo educativo equilibrado, percibido por los padres, de forma positiva $(\mathrm{r}=.15 ; \mathrm{p} \leq .05)$; $\mathrm{y}$ con el estilo educativo permisivo, percibido por los padres, de forma negativa $(\mathrm{r}=-.11 ; \mathrm{p} \leq .05)$

d) El nivel de autoestima, medido de forma global, sumando las valoraciones de la familia y escuela a partir de la observación conductual, correlaciona con el estilo educativo equilibrado, percibido por los padres $(\mathrm{r}=.22 ; \mathrm{p} \leq .05)$.

Sin embargo, en las familias de nivel sociocultural-alto no se encontraron correlaciones significativas entre los diferentes estilos educativos, tanto desde la percepción infantil como adulta, excepto $(r=.40 ; \mathrm{p} \leq .05)$ en el caso de la autoestima valorada a través de la observación conductual desde la escuela y el estilo educativo permisivo percibido por la madre.

En ambos estudios se observa una correlación negativa entre el nivel sociocultural y el estilo educativo permisivo. En las familias de nivel socio cultural más alto se identifica una correlación negativa entre el nivel de estudios del padre y la madre en el estilo permisivo familiar (padre: $r=-.20 ; \mathrm{p} \leq .001$ ) (madre: $\mathrm{r}=-.18$; $\mathrm{p} \leq .004$ ). En cambio, en las familias de nivel sociocultural más bajo, se detecta una asociación negativa entre el nivel de estudios de los padres y la percepción de los padres de su estilo educativo autoritario $(\mathrm{r}=.-21 ; \mathrm{p} \leq .01)$ y permisivo $(\mathrm{r}=.-27 ; \mathrm{p} \leq .001)$.

La edad de padres y madres es una variable moduladora en las familias de nivel sociocultural-bajo. Los datos indican que a mayor edad aumenta la permisividad de la familia: El estilo permisivo correlaciona de forma significativa y positiva con la edad del padre $(\mathrm{r}=.21 ; \mathrm{p} \leq .01)$ y la de la madre $(\mathrm{r}=.19 ; \mathrm{p} \leq .03)$. A mayor edad de los niños $\mathrm{y}$ niñas, mayor percepción del autoritarismo en la familia. Dicho de otro modo, las prácticas educativas más autoritarias son más vivenciadas cuánto mayores son los hijos e hijas.

El segundo estilo - o conjunto integrado de prácticas educativas - que las familias creen que utilizan con sus hijos es el autoritario, en lugar del permisivo, que era el utilizado por padres y madres del nivel sociocultural-alto. Por lo tanto, el conjunto de prácticas - o estilo - que creen utilizar menos las familias de nivel sociocultural-alto, es el autoritario y en las familias de nivel sociocultural-bajo, el permisivo (figuras 3 y 4 ).

No sólo es el estilo permisivo el segundo utilizado en las familias de nivel sociocultural-alto, sino que también muestra efectos diferentes. En los hijos correlaciona de 
forma positiva con la autopercepción del niño. Y la valoración de las maestras, de la expresión conductual del nivel de autoestima del niño, correlaciona o está asociado de forma negativa con la permisividad percibida por la madre $(\mathrm{r}=-.04 ; \mathrm{p} \leq .05)$.

En las familias de nivel sociocultural-bajo, el estilo permisivo sólo correlaciona de forma positiva con la edad del padre y de la madre: $(\mathrm{r}=.21 ; \mathrm{p} \leq .01)$ y la de la madre $(\mathrm{r}=.19 ; \mathrm{p} \leq .03)$. Los análisis de regresión muestran de forma consistente una relación negativa entre este estilo educativo y el nivel de autoestima del hijo (medido mediante autoinforme).

(b) Nivel sociocultural de las familias y autoestima de los hijos.

En la exploración de la autoestima se observan prácticamente los mismos resultados en los dos niveles socioculturales, tanto si se mide la autoestima con el PAI (autoinforme) como con la BSECH (observación conductual). La valoración de la familia es ligeramente superior a la valoración de la escuela. Además, coinciden ambas medidas de la autoestima, en la distribución de puntuación en las diferentes conductas representativas de los niños y en las valoraciones máximas y mínimas (figura 6).

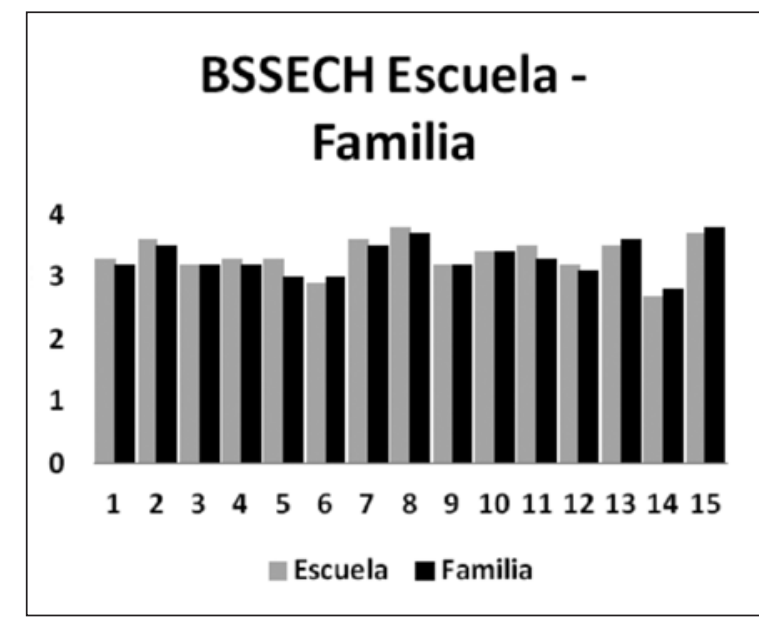

FIGURA 6

MAESTROS VS PADRES: COMPARACIÓN DE LA PERCEPCIÓN DEL NIVEL DE AUTOESTIMA DEL NIÑO EN LOS 15 ITEMS DEL BSECH (OBSERVACIÓN CONDUCTUAL). PUNTUACIÓN MÍNIMA 1, MÁXIMA 4.

Madres y maestras, de ambos niveles socioculturales, coinciden en la escasa presencia de conductas observables, en sus hijos y alumnos, del indicador: tolerancia a la frustración.

Así mismo, en ambas muestras de familias, especialmente en las familias del nivel sociocultural-bajo, el análisis factorial agrupa, por un lado, ítems con conductas representativas de niveles altos de autoestima y, por otro lado, los ítems conductas prototípicas de baja autoestima. Sin embargo hay que hacer alusión a las diferencias encontradas entre ambos niveles socioculturales: 
a) En el bajo, la autopercepción de la autoestima resulta algo más dispersa, y la hétero-percepción ha mostrado una delimitación más clara en su factorización.

b) En las percepciones de conductas consideradas como más frecuentes por padres, madres y maestras, también hay discrepancias: a pesar de coincidir ambas muestras de familias en el orgullo por sus éxitos, las familias de nivel sociocultual-bajo consideran, también, muy frecuente el interés por lo nuevo y hay pequeñas diferencias entre escuela y familia. Las maestras valoran mucho la participación en grupo, y la familia coincide con el nivel sociocultural-alto, al considerar también muy frecuente la expresión de alegría en los niños.

c) La autopercepción del niño contempla casi idénticas máximas y mínimas en los dos niveles socio-culturales. No obstante, es menester llamar la atención sobre algunas diferencias en las valoraciones mínimas. En la muestra de nivel-alto se presenta como muy poco representativo en los niños y niñas la participación en las tareas de la casa y el uso restrictivo de sus padres de amonestaciones, riñas y castigos. Las percepciones infantiles de los hijos e hijas de nivel-bajo, esos mismos aspectos son considerados como frecuentes; y entre los menos representativos de sus hogares indican el tiempo de juego compartido con sus padres y la frecuencia de regalos familiares.

\section{Discusión}

En las prácticas educativas de las familias de nivel-alto, se han encontrado los mayores niveles de acuerdo intrafamiliar. Se puede plantear la duda de si la influencia del grupo social es más impactante, o si la comunicación en las interacciones familiares es más explícita y consensuada. El estudio de acuerdos y discrepancias intrafamiliares debería ser analizado también desde otras variables, otras edades y otras percepciones (Gimeno, Córdoba, Meléndez \& Cerviño, 2004), y desde diferentes marcos comunicativos como han sugerido Gimeno, Anguera, Berzosa \& Ramírez (2006).

En las prácticas educativas de las familias de nivel-bajo, se observan más diferencias entre las percepciones de los estilos educativos que tienen hijos, padres y maestras. Las mayores discrepancias se encuentran en aquellos puntos donde el niño o niña parece necesitar mayor comprensión y sensibilidad de sus padres. La atribución infantil y legibilidad del mensaje parental dependen (a) de la anticipación con que el hijo o hija - en función de las experiencias acumuladas - realiza las inferencias respecto a las metas y estrategias de sus padres (Rodrigo \& Triana, 1996) y, (b) especialmente, las necesidades y circunstancias individuales, algo que desgraciadamente no siempre coincidentes con la actitud adulta (Grusec \& Goodnow, 1994).

En las prácticas educativas de las familias de nivel-bajo, se observa, además, la inconsistencia de las percepciones infantiles, valorando indistintamente diferentes tipos de prácticas educativas, a menudo, consideradas opuestas. Los observadores externos coinciden con la visión inconsistente y a veces extrema de los hijos con respecto al estilo educativo familiar. El estilo equilibrado o autorizador, se distribuye de forma inconsistente a lo largo de todas las situaciones y conflictos estudiados; y de forma, muchas, veces contradictoria e impredecible. Esta forma inconsistente de comportamiento de los padres hace dudar a los niños sobre la respuesta que padres y madres darán en cada 
situación conflictiva. La inconsistencia parental parece estar mediatizada por factores que inciden en el microsistema familiar - en general - y - más específicamente - en la interacción paterno y materno-filial.

En las prácticas educativas autoritarias y permisivas, hay otra diferencia entre ambas muestras de familias. En las familias nivel-bajo, el estilo permisivo correlaciona siempre de forma negativa con el estilo autoritario en padres e hijos. En cambio en el nivel-alto, hay correlación positiva (valoración realizada por los propios padres). Se confirman también diferencias en la variabilidad de las respuestas de los padres y madres en función del tipo de infracción, de las características individuales del hijo y del contexto de cada interacción, tal como han aportado investigaciones anteriores (Hoffman, 1994; Maccoby \& Martín, 1983; Musitu, Román \& Gracia, 1988; Torío, Vicente \& Inda, 2008), aportando evidencia de la bidireccionalidad o determinismo recíproco.

En función de los tipos de conflicto se constatan también, distintos niveles de acuerdo y discrepancia entre las percepciones de padres y madres e hijos e hijas. Al contrastar estos resultados con otras investigaciones, se encuentra alguna diferencia, como en el caso del nivel de acuerdo de los conflictos internos del trabajo de Ceballos, Janssens \& Rodrigo (2001). Diferente atribución intencional en las percepciones de los hijos e hijas en función del tipo de disciplina y práctica educativa parental utilizadas. El tipo de transgresión marca las expectativas de los hijos y anticipa el tipo de disciplina, coincidiendo con los resultados de las investigaciones - ya clásicas - de Siegal \& Barclay (1985) y de Tisak (1986). Los datos comparados de los dos trabajos sugieren la diferente percepción de los hijos según el tipo de conflicto y clima familiar. Hay estudios, incluso, que han detectado diferencias perceptivas según sea el tipo y estructura del contexto familiar. Además, se han encontrado resultados diferentes a los de Ceballos, Janssens \& Rodrigo $(1999,2001)$ con respecto a los conflictos relativos a la externalización y la percepción infantil de la conducta parental.

La aceptación de los mensajes paternos por parte de los hijos e hijas es variable. Su evolución y variabilidad no sólo muestra dependencia en función de los factores citados, sino que tienden a modificarse, flexibilizarse o interiorizarse, según la edad del niño, tal como aportó, y nos gustaría comprobar mejor en estudios posteriores, el trabajo de Gralinski \& Kopp (1993). Las comparaciones realizadas apuntan hacia un distanciamiento del modelo de socialización tradicional. De alguna manera, rompe con la idea de coherencia y estabilidad transituacional, encontrando, como ya se ha visto, diferentes prácticas educativas, en función de diversos factores y avalando la idea de la inexistencia de "padres puros" con estilos educativos únicos; lo que coincide con los trabajos de Triana (1991) entre otros. Los datos recogidos en este artículo apoyan, además, la frágil nitidez de los mensajes parentales, así como el diferente impacto en los hijos, en función de las características individuales del niño o niña confirmando y asumiendo, pues, la contextualización (determinismo recíproco / bidireccionalidad) planteada.

En la valoración de la autoestima infantil - con el cuestionario de autopercepciónen familias de nivel sociocultural-bajo, el ítem relacionado con los regalos no debería adoptarse como determinante del nivel de autoestima infantil, ya que, tal como se observa en su percepción de las posesiones, al realizar la comparación con los demás hogares de su entorno más próximo, no es inferior a lo encontrado en las percepciones de los niños de nivel sociocultural-alto. Tienen buena percepción de sus posesiones a pesar de valorar negativamente el ítem referente a los regalos familiares. Cabe pregun- 
tarse si ocurriría lo mismo si en ambos se hubiera descrito la historia en los mismos términos, es decir, valorando su percepción global en lugar de prefijar la frecuencia. En las familias de nivel-bajo, además, hay que prestar atención a la diferencia cultural y económica, lo cual mediatiza la interpretación de las historias descritas en cada uno de los ítems y, por tanto, el resultado global de su valoración de la autoestima.

En la muestra utilizada hay ejemplos que no son pertinentes para el grupo sociocultural al que pertenecen o la zona geográfica donde viven. Por ejemplo, citar la playa como opción es frecuentemente inutilizable por no haber estado nunca o rara vez en ella. Ayudar en casa depende, no tanto del nivel de autoestima, sino de los hábitos familiares. En las familias de nivel-bajo la colaboración desde muy pequeños es más habitual que en los niveles socioculturales más altos.

El tiempo que pasan y juegan sus padres con ellos (actividades significativas realizadas en común) depende también de sus circunstancias familiares $\mathrm{y}$, sobre todo laborales, que en las familias de nivel-bajo se reduce normalmente mucho en comparación con las de nivel-medio. Habría que seguir investigando su relación con los contextos familiares de nivel-alto y muy altos. No obstante, se pudo observar también la presencia de familias que, a pesar de contar con muy poco tiempo libre, mantenían un alto nivel de comunicación y expresión de afecto con sus hijos e hijas.

La valoración conductual de la autoestima - realizada con la escala de hétero-observación conductual - presenta una factorización que delimita claramente, por un lado, los ítems con conductas representativas de niveles altos de autoestima y, por otro lado, los ítems con conductas prototípicas de baja autoestima. Esto hace pensar en la alta probabilidad de la influencia en la elección y valoración de las respuestas en función de su presentación. Además, aunque los resultados muestran diferentes índices de frecuencia en distintos ítems, es posible que estén valorando dimensiones similares: la "falta de confianza en sí mismo", el "miedo a los retos" y la escasa preferencia por "actividades que desplieguen sus habilidades". Es posible que estén siendo el reflejo de la percepción de padres y profesores, de la "falta de confianza en sí mismo" de los hijos/alumnos.

Se aporta evidencia correlacional que confirma la importante influencia de los valores del grupo social al que pertenece la familia. Puede observarse en las elecciones de los padres y madres del estilo educativo más valorado socialmente. Parece confirmarse la influencia cultural y su diferencia transcultural, no solo en el conjunto de prácticas educativas predominantes, sino también en su consecuente influencia en el autoconcepto infantil. Estos datos apoyan la idea de Rodrigo \& Triana (1996) sobre la elaboración de las teorías implícitas de los padres y su proyección en su estilo disciplinario y actividades cotidianas con los hijos, así como su explicitación en el alto nivel de coherencia, de los padres y madres, en las elecciones y preferencias, del trabajo de Triana (1991). Sin embargo, se muestran resultados diferentes a los obtenidos en el trabajo de Rodrigo \& Triana (1996) en cuanto al estudio de la predictibilidad inferencial de las respuestas de los padres y madres con familias de un nivel sociocultural distinto al utilizado por ellas.

Las observaciones recogidas apuntan hacia la necesidad de presentar textos muy simples (las situaciones hipotéticas de las PEF), exclusivamente conductuales, con fórmulas abreviadas y genéricas, cuando se trata de trabajar con familias de nivel socioculturalbajo. Sin embargo, se ha observado también que, a medida que desciende el nivel de la muestra, el tipo de presentación exclusivamente conductual favorece el sesgo situacio- 
nal, limitándose a un exceso de concreción y mediatizando sus respuestas en función exclusivamente de la situación que se les presenta, y, por lo tanto, con más dificultades para descontextualizar, al igual que ocurre con los niños pequeños.

La conclusión principal de este estudio comparativo de los datos de dos trabajos - replicación directa uno del otro - sobre el grado de asociación o correlación entre nivel sociocultural, prácticas educativas familiares y autoestima en niños pequeños es que el nivel sociocultural correlaciona positivamente con el estilo educativo familiar autorizador o equilibrado, y éste, a su vez, con el nivel de autoestima mayor; el cual, tal como indica la evidencia empírica, repercute en diferentes dimensiones de desarrollo: ajuste emocional, éxito social y académico (García \& Gracia, 2010; Ortiz, Apodaca, Etxebarría, Fuentes \& López, 2011). De lo anterior se infiere la necesidad de promover investigaciones que faciliten el conocimiento de los entramados interactivos (número, duración e intensidad de las interacciones en los microsistemas familiar y escolar; número, duración e intensidad de las actividades significativas realizadas en común; grado de calidad en el desempeño de los roles de los padres) (Triana \& Rodríguez, 2005) en todos los niveles socioculturales, pero sobre todo en los bajos, para poder así delimitar con mayor rigor y eficacia las bases de los programas de asesoramiento familiar y de los programas de intervención psico-socio-familiar.

\section{REFERENCIAS BIBLIOGRÁFICAS}

Alonso, J., \& Román, J. M. (2003). PEF: Escalas de identificación de prácticas educativas familiares. Madrid: Cepe.

Alonso, J., \& Román, J. M. (2005a). Prácticas educativas familiares y autoestima. Psicothema, 17, 76-82.

Alonso, J., \& Román, J. M. (2005b). Estilos educativos familiares y autoestima en los hijos de 4 y 5 años. Psicología General y Aplicada, 58(1), 101-104.

Baumrind, D. (1971). Current patterns of parental authority. Developmental Psychology Monograph, 4, 1-103.

Calvete, E., Orue, I., \& Sampedro, R. (2011). Violencia filio-parental en la adolescencia: características ambientales y personales. Infancia y Aprendizaje, 34(3), 349-363.

Ceballos, E .M., Janssens, J. M., \& Rodrigo, M. J. (1999). Do children's perceptions and attributions mediate the effects of mother's child-rearing actions? Journal of Family Psychology, 13(4), 508-522.

Ceballos, E. M., Janssens, J. M., \& Rodrigo, M. J. (2001). Reasoning and action complexity: Sources and consequences on maternal child-rearing behaviour. International Journal of Behavioral Development, 25(1), 50-59.

Fraguela, R., Lorenzo, J. J., \& Varela, L. (2011). Escuela, familias y ocio en la conciliación de los tiempos cotidianos de la infancia. Revista de Investigación Educativa, 29(2), 429-446.

García, F., \& Gracia, E. (2010). ¿Qué estilo de socialización parental es el idóneo en España? Un estudio con niños y adolescentes de 10 a 14 años. Infancia y Aprendizaje, 33(3), 365-384.

Gimeno, A., Córdoba, A. I., Meléndez, J. C., \& Cerviño, C. (2004). Divergencias en la percepción de la funcionalidad familiar entre padres, madres e hijos adolescentes. Revista Latinoamericana de Psicología, 36(3), 85-100. 
Gimeno, A., Anguera, T., Berzosa, A., \& Ramírez, L. (2006). Detección de patrones interactivos en la comunicación de familias con hijos adolescentes. Psicothema, 18, 785-790.

Gralinski, J .H., \& Kopp, C. B. (1993). Everyday rules for behavior: Mothers requests to young children. Developmental Psychology, 29(3), 573-584.

Grusec, J. E., \& Goodnow, J. J. (1994). Impact of parental discipline methods on the Child's Internalization of Values: A reconceptualization of current points of view. Developmental Psychology, 30, 4-19.

Haltiwanger, J., \& Harter, S. (1988). The behavioral rating scale of presented self-esteem in young children. Denver: Universidad de Denver.

Hoffman, M. L. (1994). Discipline and internalization. Development Psychology, 30(1), 26-28.

Maccoby, E. E., \& Martín, J .A. (1983). Socialization in the context of the family: Parentchild interaction. En P. H. Mussen \& E. M. Hetherington (Eds.), Handbook of child psychology. Socialization, personality and social development (4⿳亠丷厂 ed., Vol. 4, pp.1-102). Nueva York: Wiley.

Musitu, G., Román, J. M., \& Gracia, E. (1988). Familia y Educación. Prácticas educativas de los padres y socialización de los hijos. Barcelona: Labor.

Ondersma, S. J. (2002). Predictors of neglect within low-SES families: The importance of substance abuse. American Journal of Orthpsychiatry. 72, 383.391.

Ortiz, M. J., Apodaca, P., Etxebarria, I., Fuentes, M .J., \& López, F. (2011). Papel de los padres y madres en la regulación moral de los niños y en la conducta prosocial y agresiva con los compañeros. Infancia y Aprendizaje, 34(3), 365-380.

Rodrigo, M .J., \& Triana, B. (1996). Parental beliefs about child development and parental inferences about actions during child -rearing episodes-. European Journal of Psychology, 11(1), 55-78.

Siegal, M., \& Barclay, M. S. (1985). Children's evalutions of father's socialization behavior. Developmental Psychology, 21(6), 1090-1096.

Soriano, E., \& Franco, C. (2011). Mejora de la autoestima y de competencia emocional en adolescentes inmigrantes sudamericanos a través de un programa psicoeducativo de "mindfulness". Revista de Investigación Educativa, 28(2), 297-312.

Tisak, M. S. (1986). Children's conceptions of parental authority. Child Development, $57,166-176$.

Torío, S., Vicente, J., \& Inda, M. (2008). Estilos de educación familiar. Psicothema, 20, 62-70.

Triana, B. (1991). Las concepciones de los padres sobre el desarrollo: Teorías personales o teorías culturales. Infancia y Aprendizaje, 54, 19-39.

Triana, B., \& Rodriguez, G. (2005). La experiencia familiar y la atribución de roles parentales. Psicothema, 17, 363-369.

Villa, A., \& Auzmendi, E. (1999). Desarrollo y evaluación del autoconcepto en la edad infantil. Bilbao: Mensajero.

Weiland, C., \& Yoshikawa, H. (2012). The effects of large-scale economic change and policies on children's developmental contexts and developmental outcomes. Child Development Perspectives, 6, 342-350.

Fecha de recepción: 27 de octubre de 2012.

Fecha de revisión: 10 de noviembre de 2012.

Fecha de aceptación: 29 de junio de 2013. 